\title{
Biomarkers for the prediction of acute ongoing arterial plaque rupture
}

This article was published in the following Dove Press journal:

Research Reports in Clinical Cardiology

II July 2013

Number of times this article has been viewed

\section{Yuan-Lin Guo \\ Jian-Jun Li}

Division of Dyslipidemia, State Key Laboratory of Cardiovascular Disease, Fu Wai Hospital, National Center for Cardiovascular Disease, Chinese Academy of Medical Sciences and Peking Union Medical College, Beijing, People's Republic of China
Correspondence: Jian-Jun Li Division of Dyslipidemia, State Key Laboratory of Cardiovascular Disease, Fu Wai Hospital, National Center for Cardiovascular Disease, Chinese Academy of Medical Sciences and Peking Union Medical College, Beijing, I00037,

People's Republic of China

Tel +861088396077

Fax +86I0683 3I730

Email lijnjn@yahoo.com.cn
Abstract: Acute coronary syndrome (ACS) is the main cause of mortality for coronary artery disease (CAD). Accordingly, earlier detection and diagnosis might be a key point for reducing the mortality in patients with ACS. One promising strategy is biomarker measurement in patients with ACS. Biomarkers are generally considered to be plasma measurements of molecules, proteins, or enzymes that provide independent diagnostic and prognostic values that can reflect underlying disease state and condition, especially repeated measurements. Nowadays, the most widely used biomarkers to identify or predict ACS are high sensitivity C-reactive protein (hsCRP) and high sensitivity troponin T/I (hs-TnT/I). The aim of the present review was principally to summarize recent evidence regarding some new biomarkers by which we could directly predict acute ongoing arterial plaque rupture, which may help to identify at-risk patients earlier than hs-CRP or hs-TnT/I.

Keywords: matrix metalloproteinase-9, lipoprotein associated phospholipase A2, myeloperoxidase, soluble lectin-like oxidized low-density lipoprotein receptor-1, pregnancyassociated plasma protein A, placental growth factor, acute coronary syndrome

\section{Introduction}

It is well-recognized that the pivotal pathophysiologic mechanism of acute coronary syndrome (ACS) is an unstable atherosclerotic plaque rupture with following thrombosis. ${ }^{1}$ However, only by intravascular imaging technology or biopsy/autopsy of atherosclerotic plaques can these processes be directly identified. Additionally, it is often too late to prevent cardiac death at that time. Moreover, many patients with unstable angina were found to have no serious stenosis in coronary artery by angiography. Accordingly, these patients were not suitable to be detected and treated by the method of coronary artery angiography and intervention. For these cases with unstable clinical symptoms, one of the easiest new approaches that could be incorporated into clinical practice would be to test a peripheral blood sample for the presence of a specific atherosclerosis marker. Up to now, the early diagnosis of ACS is still challenging because of its various nonspecific clinical manifestations. However, biomarkers in blood circulation have been gaining cardiologists' interest in recent years. ${ }^{2}$

An ideal biomarker should be directly related to plaque rupture, appear very early in circulation, and be able to be detected at the bedside quickly and easily. Biomarkers with these characteristics will help us to identify patients at risk of ACS as early as we can. Nowadays, one of the most widely used biomarkers to identify ACS is high sensitivity troponin T/I (hs-TnT/I) and for ACS risk stratification is high sensitivity 
C-reactive protein (hs-CRP). A rich body of evidence has been produced in the last two decades about the predictable values of hs-TnT/I and hs-CRP for ACS, especially in a repeated measurement manner. ${ }^{3,4}$ There are many other biomarkers reflecting different mechanisms clinically available for predicting adverse cardiovascular events; for example, inflammation-related, hemostasis- and thrombosisrelated, oxidative stress-related, myocardial damage- and tissue necrosis-related biomarkers. This review, however, is principally aimed at summarizing recent evidence regarding potential new biomarkers which could directly predict acute ongoing arterial plaque rupture (Table 1).

\section{Matrix metalloproteinase-9}

Matrix metalloproteinase-9 (MMP-9) is regarded as a biomarker of plaque rupture or vulnerability, and several studies recently demonstrated that serum MMP-9 level elevation might be a significant new biomarker of ACS. ${ }^{5-9}$ A study from a Japanese population demonstrated that plasma MMP-9 was an independent predictor of plaque rupture assessed by intravascular ultrasound of culprit lesion in patients with acute myocardial infarction (MI) or unstable angina, even after adjustment for the traditional risk factors. ${ }^{10}$ Wang et $\mathrm{al}^{11}$ further revealed that circulating MMP-9 could help differentiate ACS patients with plaques from those without plaques. Because MMP-9 was directly related to atherosclerotic plaque rather than myocardial necrosis, it may have specific high value in the early identification of ruptured plaque. This hypothesis was recently verified in a study performed by Kobayashi et al. ${ }^{12}$ They measured MMP-9 and hs-TnT in 200 patients with ST-elevated ACS (115 early ST-elevated ACS; 85 late ST-elevated ACS patients), and 66 patients with non-ST elevated ACS (25 early non-ST elevated ACS; 41 late non-ST elevated ACS patients). An additional 40 patients with stable angina pectoris were enrolled as a control group. The results showed that MMP-9 levels were elevated earlier than hs-TnT and also had a higher diagnostic value for early ACS ( $<4$ hours), but not for late ACS. ${ }^{12} \mathrm{CRP}$ is currently known as an independent risk factor for cardiovascular events. Regarding the relationship between MMP-9 and CRP, a recent study found that the CRP dose dependent-induced expression of MMP-9 in smooth muscle cells in vitro and the local production of CRP in patients with ACS related to an increase in transcoronary MMP-9 levels. ${ }^{13}$ These results indicated that CRP might exert a causative role in plaque complication in vivo through induction and release of MMP-9 in the coronary circulation of ACS patients.

In summary, the elevation of MMP-9 reflects plaque rupture or vulnerability and precedes that of biomarkers for myocardial damage, because plaque rupture or vulnerability precedes myocardial damage in ACS. Recent evidence strongly indicates that the early diagnostic value of MMP-9 might be superior to that of hs-TnT, and the predictive value of MMP-9 might be equal or superior to that of CRP in patients with ACS.

\section{Lipoprotein associated phospholipase A2}

The lipoprotein-associated phospholipase A2 (Lp-PLA $)_{2}$, a 45.4-kDa protein, is a calcium-independent member of the phospholipase A2 family. It is produced mainly by monocytes, macrophages, T-lymphocytes, and mast cells, and has been found to be upregulated in atherosclerotic lesions, especially in complex plaque, as well as in the thin cap coronary lesions prone to rupture. Functionally, Lp-PLA $\mathrm{A}_{2}$ has proatherogenic properties, mainly by promoting oxidation of low-density lipoprotein (LDL). ${ }^{14,15}$ Several studies have found an association between increased concentrations of Lp-PLA $\mathrm{A}_{2}$ and future coronary and cerebrovascular events, independent of a variety of potential confounders. ${ }^{16-19}$ A study by Kolodgie et $\mathrm{al}^{20}$ showed that Lp-PLA 2 was strongly expressed within the necrotic core and surrounding

Table I Current and potential new biomarkers in ACS

\begin{tabular}{|c|c|c|c|c|}
\hline \multirow{2}{*}{$\begin{array}{l}\text { Early stage } \\
\text { Plaque's } \\
\text { cell composition- } \\
\text { related }\end{array}$} & \multicolumn{2}{|l|}{ Early-to-late stage } & \multicolumn{2}{|l|}{ Late stage } \\
\hline & $\begin{array}{l}\text { Oxidative stress- } \\
\text { related }\end{array}$ & $\begin{array}{l}\text { Inflammation- } \\
\text { related }\end{array}$ & $\begin{array}{l}\text { Platelet activation- } \\
\text { related }\end{array}$ & $\begin{array}{l}\text { Tissue damage- } \\
\text { related }\end{array}$ \\
\hline Matrix metalloproteinase-9 & Myeloperoxidase & hs-CRP & Soluble CD40 ligand & Troponin \\
\hline $\begin{array}{l}\text { Pregnancy-associated plasma } \\
\text { protein A }\end{array}$ & $\begin{array}{l}\text { Lipoprotein-associated } \\
\text { phospholipase A2 }\end{array}$ & Interleukin-6 & Soluble P-selectin & $\begin{array}{l}\text { Heart fatty } \\
\text { acid-binding protein }\end{array}$ \\
\hline Placental growth factor & $\begin{array}{l}\text { Soluble lectin-like oxidized low- } \\
\text { density lipoprotein receptor-I }\end{array}$ & $\begin{array}{l}\text { Intracellular adhesion } \\
\text { molecule I } \\
\text { Tumor necrosis factor- } \alpha\end{array}$ & & \\
\hline
\end{tabular}

Abbreviations: ACS, acute coronary syndrome; hs-CRP, high sensitivity C-reactive protein. 
macrophages of vulnerable and ruptured plaques, with relatively weak staining in less-advanced lesions. These findings, together with the association of Lp-PLA $\mathrm{L}_{2}$ in apoptotic macrophages, suggest a potential role in promoting plaque instability. More recently, a study performed by Liu et $\mathrm{al}^{21}$ measured plasma Lp-PLA 2 activity in 146 consecutive patients with coronary artery disease (CAD) who underwent clinical-indicated coronary angiography and preinterventional intravascular ultrasound (IVUS). Their results further demonstrated that plasma Lp-PLA 2 activity was associated with plaque rupture, independently of traditional risk factors, such as hs-CRP level and IVUS parameters in patients with CAD. In addition, inhibiting Lp-PLA 2 by its direct inhibitor darapladib could also represent an attractive novel therapeutic target to stabilize atherosclerotic plaque by limiting the progress of its lipid core, which has been demonstrated by a study from Serruys et al. ${ }^{22}$ So, Lp-PLA 2 may be a reliable risk marker for vulnerable plaque. However, it has been reported that plasma concentration of Lp-PLA is also increased evidently in stable CAD. ${ }^{23}$ Hence, its use as a marker of plaque rupture would require more evidence or regularly repeated measurements to define an exact and reliable level for clinical diagnosis or the prediction of unstable plaques rather than stable plaques.

\section{Myeloperoxidase}

Oxidative stress participates in all stages of atherosclerosis, from lipoprotein modification to plaque rupture; biomarkers of oxidative stress, such as myeloperoxidase (MPO), may predict the vulnerability of atherosclerotic plaque.

MPO is an enzyme found primarily in neutrophils and monocytes. It is identified in large quantities in human vulnerable atherosclerotic plaque and exerts potent proatherogenic and proinflammatory effects. ${ }^{24}$ MPO leads to plaque rupture through several pathways, including lipid oxidation, endothelial dysfunction, MMP activation, and collagen layer degradation, etc. ${ }^{25}$

Many studies have found evidence of elevated MPO levels in ACS patients. The C7e3 fab AntiPlatelet Therapy in Unstable REfractory angina (CAPTURE) trial ${ }^{2}$ investigators demonstrated that serum MPO levels were associated with prognosis of cardiovascular death or recurrent $\mathrm{MI}$ in a sample of 1090 patients with an ACS event. MPO was the strongest predictor (hazard ratio [HR]: 2.11) of adverse events in a 6-month follow-up period compared to troponin, CRP, vascular endothelial growth factor (VEGF), or soluble CD40 ligand (CD40 L) and was especially capable of predicting such events in the cohort of troponin-negative patients (HR: 7.48). 2,26 Recently, another study further suggested the predictive value of MPO among patients without troponin elevation. That study showed that a single measurement of the plasma MPO concentration could help identify patients with a higher risk of having significant coronary stenoses and high-risk angiographic features. ${ }^{27}$ In addition, MPO may offer a long-term predictive value for cardiovascular risks. To make sure of this result, Heslop et $\mathrm{al}^{28}$ measured MPO, nitrotyrosine, oxidized lowdensity lipoprotein, and antioxidant capacity in a prospective cohort of 885 selective coronary angiography patients, and followed up for $>13$ years for cardiovascular mortality. The results showed MPO accurately predicted cardiovascular mortality risk in coronary angiography patients. Accordingly, combination MPO and CRP together may improve long-term risk assessment and $\mathrm{CAD}$ patient outcomes. ${ }^{28}$ Moreover, some studies reported that MPO levels were elevated only among ACS patients. In contrast, CRP levels increased in both ACS and stable patients, ${ }^{29}$ suggesting that MPO may be more correlated to ongoing plaque rupture while CRP may be more useful for predicting long-term cardiac events among those who recently presented with stable angina. ${ }^{23,29}$ In other words, elevated MPO levels among the ACS patients suggest that this marker may participate in plaque vulnerability and the instability process, whereas higher CRP levels were predictive of cardiac events only among the stable angina patients.

The above data suggest that MPO might be a promising biomarker for the detection of ongoing ruptured plaque and myocardial ischemia, as well as for the risk stratification in patients with ACS. However, MPO determination currently lacks standardized analytical and preanalytical methods. Additionally, therapeutic doses of heparin could interfere to the plasma MPO values measured. These issues may limit the clinical application of MPO in everyday practice.

\section{Soluble lectin-like oxidized low- density lipoprotein receptor-I}

Lectin-like oxidized low-density lipoprotein receptor-1 (LOX-1) is a receptor for atherogenic oxidized lowdensity lipoprotein (ox-LDL).$^{30}$ Previous pathological study showed that LOX-1 was prominently expressed by intimal smooth muscle cells and lipid-laden macrophages in human advanced atherosclerotic plaques. ${ }^{31}$ It has already been demonstrated that LOX-1 expression was associated with the instability of atherosclerotic plaques in a hypercholesterolemic animal model.$^{32}$ As is known, ox-LDL affects plaque vulnerability through apoptosis of vascular 
endothelial and smooth muscle cells and production of MMP. However, in this process, in which it is directly linked to plaque rupture, LOX-1 plays an important and imperative role. ${ }^{33,34}$ The above data suggest that LOX-1 may affect atherosclerotic progression and plaque vulnerability through several mechanisms.

When LOX-1 is proteolytically cleaved at its membrane proximal extracellular domain, it will be released in a soluble form, as soluble LOX-1 (sLOX-1). Several studies have showed circulating sLOX-1 levels are dramatically elevated in patients with ACS. Data from Hayashida et al ${ }^{35}$ showed the excellent sensitivity and specificity of sLOX-1 for early identification of ACS. In this study, they examined serum sLOX-1 levels in 521 patients, including 80 patients with ACS, 173 symptomatic patients with CAD, 122 patients with significant coronary stenosis without ischemia, and 52 patients without apparent coronary atherosclerosis, plus 34 patients with noncardiac acute illness and 60 patients with noncardiac chronic illness.

Time-dependent changes in sLOX-1 and TnT levels were analyzed in an additional 40 ACS patients. The results showed serum sLOX-1 levels were significantly higher in ACS than that of other groups, and sLOX-1 levels were associated with ACS, as shown by multivariable logistic regression analyses. Moreover, the sensitivity and specificity of sLOX-1 were much higher compared with hs-CRP and TnT. Also, peak values of sLOX-1 in ACS were observed earlier than those of TnT. These results indicated sLOX-1 might be a more useful novel biomarker for early diagnosis of ACS, especially for early detection for those without Q wave and those without significant elevation of TnT levels at the time of visit. ${ }^{35}$ Recently, another study from Kume et $\mathrm{al}^{36}$ further demonstrated that the sensitivity and specificity of sLOX-1 for diagnosis of ACS were superior to those of TnT. In this study, the investigators compared the diagnostic value of sLOX-1 for ACS with those of TnT and heart-type fatty acid-binding protein (H-FABP) in 107 patients who underwent coronary angiography, including 18 ACS and 89 non-ACS. The results suggest that circulating sLOX-1 level is a biomarker more sensitive and specific for ACS than TnT and H-FABP, and provides additional diagnostic values when measured in combination with TnT.

In addition, a pilot study performed by Kume et $\mathrm{al}^{37}$ followed up 94 ACS patients undergoing emergency percutaneous coronary intervention for a median time of 29 months. The results showed that besides its good diagnostic value, sLOX-1 also predicted ACS recurrence or death well.
In summary, because sLOX-1 is directly linked to plaque instability, it may be a more useful marker than those biomarkers linked to myocardial ischemia or necrosis for diagnosing ACS at the earliest stage.

\section{Pregnancy-associated plasma protein-A}

The pregnancy-associated plasma protein-A (PAPP-A) is a high molecular mass metalloproteinase. It is so named because of its special use as a prenatal screening test for the detection of trisomy 21 (Down syndrome) during the first trimester of pregnancy. ${ }^{38}$ Previous studies showed that PAPP-A might be produced by different activated cells in unstable atherosclerotic plaques, released into the extracellular matrix in the artery wall, and circulated in a form of a heterotetrameric complex. Using specific monoclonal antibodies, PAPP-A was found to be abundantly expressed in both eroded and ruptured coronary and carotid plaques, mainly in monocyte/macrophages present in the cap and shoulder region in vulnerable plaque. ${ }^{39}$ Functionally, PAPP-A is considered to be participating in many local proliferative processes, including the healing of atherosclerotic plaque which thus affects the vulnerability of plaques. However, it is still uncertain whether PAPP-A can directly degrade extracellular matrix.

A large number of studies have suggested that circulating PAPP-A levels were significantly higher in patients with ACS and that its high value of predicting cardiac events was independent of troponin. ${ }^{40-45}$ Of those, the most representative one was the CAPTURE trial that showed that high PAPP-A levels were related to an increased risk of death and $\mathrm{MI}$, in both patients with troponin negative or positive tests. In multivariable analyses, PAPP-A, soluble CD40 ligand (sCD40L), interleukin 10 (IL-10), and VEGF were independent predictors of outcome. ${ }^{45} \mathrm{~A}$ recent study which enrolled ACS patients with elevated cardiac enzyme levels $(n=105)$ and with normal cardiac enzyme profiles $(n=62)$ showed similar efficacies of CRP and PAPP-A levels in their ability to detect unstable plaques. The results highlight the utility of both CRP and PAPP-A levels as determinants of plaque instability. ${ }^{46}$

It seemed that PAPP-A might be a very attractive novel biomarker for identifying plaque rupture before a detectable increasing in cardiac troponin $\mathrm{T}$ and for predicting future outcome in patients with acute chest pain. However, further studies are needed to make PAPP-A easy and accurate enough to be used clinically at emergency departments. 


\section{Placental growth factor}

Placental growth factor (PlGF) is another important novel candidate biomarker of plaque instability. It is a heterodimeric angiogenic protein belonging to the VEGF superfamily. PIGF can be abundantly secreted by various types of cells, such as vascular smooth muscle cells and inflammation-mediated cells, under the condition that cells are activated by different kinds of stresses. ${ }^{47}$ There is evidence that a high-level PlGF plays an important role in plaque development, destabilization, and rupture by increasing proliferation and migration of endothelial cells and vascular smooth muscle cells, chemotactic recruitment of circulating monocytes and macrophages into atherosclerotic lesions, and the upregulation of several cytokines that promote plaque progress. ${ }^{48,49}$

Also, evidence from several clinical trials indicated that circulating PlGF could be a good biomarker and predictor for ACS. In the CAPTURE trial, elevated concentrations of PIGF at admission significantly distinguished patients with ACS from those with other benign reasons of acute chest pain. The levels were also significantly associated with an increased risk of adverse events (death or nonfatal MI) at both 30 days and 4 years. Furthermore, this association was independent of several other biomarkers, such as troponin, $\mathrm{SCD} 40 \mathrm{~L}$ and hs-CRP. ${ }^{50,51}$ Additionally, another study by Apple et al ${ }^{52}$ also demonstrated the promising prognostic value of PIGF in a heterogeneous group of 457 patients presenting with subtle symptoms of ACS. However, Möckel et al ${ }^{53}$ using a multimarker strategy, didn't find that PlGF level at admission could predict death and MI at 42 days, in a population of 432 patients admitted for a suspected ACS. The reason for this disparity may be linked to the population studied. It was considered that PIGF would have performed better in a higher risk cohort while their study population was at an overall low risk for mortality. Recently, a study from Glaser et $a l^{54}$ demonstrated the high prognostic value of PIGF by combining it with a B-type natriuretic peptide and estimated glomerular filtration rate. They considered that a combination might be the most accurate method in riskstratifying patients with suspected ACS.

The above data may suggest that PIGF is a potential useful biomarker for the early identification of ACS, by detecting ongoing plaque rupture. Further studies on its mechanism as well as its clinical practice are needed.

\section{Conclusion}

The development of promising circulating biomarkers is very attractive for clinicians. However, a single biomarker, even if it is ideal, is not enough for early identifying of an ACS, due to the complex nature of the disease. In daily clinical practice, it is much better and wiser to combine multiple classical and novel biomarkers as well as other noninvasive or invasive techniques to make a final decision. ${ }^{55-57}$

Currently, there are lots of biomarkers for predicting ACS under investigation. Biomarkers mentioned in this review are only a part of those directly related to ongoing plaque rupture, which would be released into the circulation prior to markers of myocardial damage, and thus it would be more useful for identifying an ACS at its earliest stage. It is worthy of mention that biomarkers of plaque instability do not seem helpful in the early diagnosis of acute MI, but they do provide high value in the early diagnosis of unstable angina with troponin negative, and in the risk stratification of patients with acute chest pain. ${ }^{58}$ Evidently, it is extremely important to identify patients, prior to the development of acute MI, because it would make a quite different outcome by early guidance to the right therapy strategy. In addition, sLOX1, PAPP-A, and PlGF are associated with inflammation in general and are not independent predictors of plaque rupture. Their clinical use is highly hypothetical accordingly. As for the other early markers of plaque rupture, only repeated measurements in patients at risk can detect plaque rupture. Further prospective studies are needed to verify the diagnostic and prognostic value of the above potential biomarkers used alone and in combination in the real world clinical practice of cardiovascular medicine.

\section{Acknowledgments}

This work is partly supported by the National Natural Scientific Foundation of China (81070171, 81241121), Specialized Research Fund for the Doctoral Program of Higher Education of China (20111106110013), Capital Special Foundation for Clinical Application Research (Z121107001012015), awarded to Jian-Jun Li and partly supported by the Fu Wai Hospital Youth Fund (Number 2010-F02), awarded to Yuan-Lin Guo. The financial supporters had no role in the design, conduct, analysis, or reporting of this study.

\section{Disclosure}

The authors report no conflicts of interest in this work.

\section{References}

1. Libby P. Current concepts of the pathogenesis of the acute coronary syndromes. Circulation. 2001;104(3):365-372.

2. Tousoulis D, Hatzis G, Papageorgiou N, et al. Assessment of acute coronary syndromes: focus on novel biomarkers. Curr Med Chem. 2012; 19(16):2572-2587. 
3. Ridker PM, Hennekens CH, Buring JE, Rifai N. C-reactive protein and other markers of inflammation in the prediction of cardiovascular disease in women. $N$ Engl J Med. 2000;342(12):836-843.

4. Reichlin T, Hochholzer W, Bassetti S, et al. Early diagnosis of myocardial infarction with sensitive cardiac troponin assays. $N$ Engl J Med. 2009;361(19):858-867.

5. Brown DL, Hibbs MS, Kearney M, Loushin C, Isner JM. Identification of 92-kD gelatinase in human coronary atherosclerotic lesions. Association of active enzyme synthesis with unstable angina. Circulation. 1995;91(8):2125-2131.

6. Kai H, Ikeda H, Yasukawa H, et al. Peripheral blood levels of matrix metalloproteases-2 and -9 are elevated in patients with acute coronary syndromes. J Am Coll Cardiol. 1998;32(2):368-372.

7. Konstantino Y, Nguyen TT, Wolk R, Aiello RJ, Terra SG, Fryburg DA. Potential implications of matrix metalloproteinase-9 in assessment and treatment of coronary artery disease. Biomarkers. 2009;14(2):118-129.

8. Bittner A, Alcaino H, Castro PF, et al. Matrix metalloproteinase-9 activity is associated to oxidative stress in patients with acute coronary syndrome. Int J Cardiol. 2010;143(1):98-100.

9. Park JP, Lee BK, Shim JM, et al. Relationship between multiple plasma biomarkers and vulnerable plaque determined by virtual histology intravascular ultrasound. Circ J. 2010;74(2):332-336.

10. Fukuda D, Shimada K, Tanaka A, et al. Comparison of levels of serum matrix metalloproteinase-9 in patients with acute myocardial infarction versus unstable angina pectoris versus stable angina pectoris. Am J Cardiol. 2006;97(2):175-180.

11. Wang LX, Lü SZ, Zhang WJ, Song XT, Chen H, Zhang LJ. Comparision of high sensitivity $\mathrm{C}$-reactive protein and matrix metalloproteinase-9 in patients with unstable angina between with and without significant coronary artery plaques. Chin Med J (Engl). 2011;124(11):1657-1661.

12. Kobayashi N, Hata N, Kume N, et al. Matrix metalloproteinase- 9 for the earliest stage acute coronary syndrome. Circ J. 2011;75(12): 2853-2861.

13. Cimmino G, Ragni M, Cirillo P, et al. C-reactive protein induces expression of matrix metalloproteinase-9: A possible link between inflammation and plaque rupture. In J Cardiol. Epub November 14, 2012.

14. Zalewski A, Macphee C. Role of lipoprotein-associated phospholipase A2 in atherosclerosis: biology, epidemiology, and possible therapeutic target. Arterioscler Thromb Vasc Biol. 2005;25(5):923-931.

15. Koenig W, Khuseyinova N. Biomarkers of atherosclerotic plaque instability and rupture. Arterioscler Thromb Vasc Biol. 2007;27(1): 15-26.

16. Packard CJ, O'Reilly DS, Caslake MJ, et al. Lipoprotein-associated phospholipase A2 as an independent predictor of coronary heart disease. West of Scotland Coronary Prevention Study Group. $N$ Engl J Med. 2000;343(16):1148-1155.

17. Mallat Z, Steg PG, Benessiano J, et al. Circulating secretory phospholipase A2 activity predicts recurrent events in patients with severe acute coronary syndromes. J Am Coll Cardiol. 2005;46(7):1249-1257.

18. Oei HH, van der Meer IM, Hofman A, et al. Lipoprotein-associated phospholipase A2 activity is associated with risk of coronary heart disease and ischemic stroke: the Rotterdam Study. Circulation. 2005; 111(5):570-575.

19. Koenig W, Twardella D, Brenner H, Rothenbacher D. Lipoproteinassociated phospholipase A2 predicts future cardiovascular events in patients with coronary heart disease independently of traditional risk factors, markers of inflammation, renal function, and hemodynamic stress. Arterioscler Thromb Vasc Biol. 2006;26(7):1586-1593.

20. Kolodgie FD, Burke AP, Skorija KS, et al. Lipoprotein-associated phospholipase A2 protein expression in the natural progression of human coronary atherosclerosis. Arterioscler Thromb Vasc Biol. 2006; 26(11):2523-2529.

21. Liu CF, Qin L, Ren JY, et al. Elevated plasma lipoprotein-associated phospholipase $\mathrm{A}_{2}$ activity is associated with plaque rupture in patients with coronary artery disease. Chin Med J (Engl). 2011;124(16): 2469-2473.
22. Serruys PW, García-García HM, Buszman P, et al. Effects of the direct lipoprotein-associated phospholipase A(2) inhibitor darapladib on human coronary atherosclerotic plaque. Circulation. 2008;118(11): 1172-1182.

23. Uydu HA, Bostan M, Yilmaz A, et al. Comparison of inflammatory biomarkers for detection of coronary stenosis in patients with stable coronary artery disease. Eur Rev Med Pharmacol Sci. 2013;17(1): $112-118$.

24. Singh U, Devaraj S, Jialal I. C-reactive protein stimulates myeloperoxidase release from polymorphonuclear cells and monocytes: implications for acute coronary syndromes. Clin Chem. 2009;55(2):361-364.

25. Nicholls SJ, Hazen SL. Myeloperoxidase and cardiovascular disease. Arterioscler Thromb Vasc Biol. 2005;25(6):1102-1111.

26. Baldus S, Heeschen C, Meinertz T, et al. Myeloperoxidase serum levels predict risk in patients with acute coronary syndromes. Circulation. 2003;108(12):1440-1445.

27. Rebeiz AG, Tamim HM, Sleiman RM, et al. Plasma myeloperoxidase concentration predicts the presence and severity of coronary disease in patients with chest pain and negative troponin-T. Coron Artery Dis. 2011;22(8):553-558.

28. Heslop CL, Frohlich JJ, Hill JS. Myeloperoxidase and C-reactive protein have combined utility for long-term prediction of cardiovascular mortality after coronary angiography. J Am Coll Cardiol. 2010;55(11): 1102-1109.

29. Roman RM, Camargo PV, Borges FK, Rossini AP, Polanczyk CA. Prognostic value of myeloperoxidase in coronary artery disease: comparison of unstable and stable angina patients. Coron Artery Dis. 2010;21(3):129-136.

30. Sawamura T, Kume N, Aoyama T, et al. An endothelial receptor for oxidized low-density lipoprotein. Nature. 1997;386(6620):73-77.

31. Kataoka H, Kume N, Miyamoto S, et al. Expression of lectinlike oxidized low-density lipoprotein receptor-1 in human atherosclerotic lesions. Circulation. 1999;99(24):3110-3117.

32. Ishino S, Mukai T, Kuge Y, et al. Targeting of lectinlike oxidized lowdensity lipoprotein receptor 1 (LOX-1) with 99 mTc-labeled anti-LOX-1 antibody: potential target for imaging of vulnerable plaque. $J \mathrm{Nucl} \mathrm{Med}$. 2008;49(10):1677-1685.

33. Kume N, Kita T. Apoptosis of vascular cells by oxidized LDL: involvement of caspases and LOX-1 and its implication in atherosclerotic plaque rupture. Circ Res. 2004;94(3):269-270.

34. Li D, Liu L, Chen H, Sawamura T, Ranganathan S, Mehta JL. LOX-1 mediates oxidized low-density lipoprotein-induced expression of matrix metalloproteinases in human coronary artery endothelial cells. Circulation. 2003;107(4):612-617.

35. Hayashida K, Kume N, Murase T, et al. Serum soluble lectin-like oxidized low-density lipoprotein receptor-1 levels are elevated in acute coronary syndrome: a novel marker for early diagnosis. Circulation. 2005;112(6):812-818.

36. Kume N, Mitsuoka H, Hayashida K, Tanaka M, Kominami G, Kita T. Soluble lectin-like oxidized LDL receptor-1 (sLOX-1) as a sensitive and specific biomarker for acute coronary syndrome - comparison with other biomarkers. J Cardiol. 2010;56(2):159-165.

37. Kume N, Mitsuoka H, Hayashida K, Tanaka M, Kita T. Soluble lectinlike oxidized low-density lipoprotein receptor-1 predicts prognosis after acute coronary syndrome - a pilot study. Circ J. 2010;74(7): 1399-1404.

38. Palomaki GE, Wright DE, Summers AM, et al. Repeated measurement of pregnancy-associated plasma protein-A (PAPP-A) in Down syndrome screening: a validation study. Prenat Diagn. 2006;26(8):730-739.

39. Sangiorgi G, Mauriello A, Bonanno E, et al. Pregnancy-associated plasma protein-A is markedly expressed by monocyte-macrophage cells in vulnerable and ruptured carotid atherosclerotic plaques: a link between inflammation and cerebrovascular events. J Am Coll Cardiol. 2006;47(11):2201-2211.

40. Bayes-Genis A, Conover CA, Overgaard MT, et al. Pregnancyassociated plasma protein A as a marker of acute coronary syndromes. N Engl J Med. 2001;345(14):1022-1029. 
41. You L, Li L, Zhang F, Xu Q, Ren J. A pilot study of the clinical relevance of the relationship between the serum level of pregnancy-associated plasma protein A and the degree of acute coronary syndrome. $J$ Int Med Res. 2010;38(2):625-632.

42. Lund J, Qin QP, Ilva T, et al. Circulating pregnancy-associated plasma protein A predicts outcome in patients with acute coronary syndrome but no troponin I elevation. Circulation. 2003;108(16):1924-1926.

43. Iversen KK, Teisner AS, Teisner B, et al. Pregnancy associated plasma protein A, a novel, quick, and sensitive marker in ST-elevation myocardial infarction. Am J Cardiol. 2008;101(10):1389-1394.

44. Lund J, Qin QP, Ilva T, et al. Pregnancy-associated plasma protein A: a biomarker in acute ST-elevation myocardial infarction (STEMI). Ann Med. 2006;38(3):221-228.

45. Heeschen C, Dimmeler S, Hamm CW, et al. Pregnancy-associated plasma protein-A levels in patients with acute coronary syndromes: comparison with markers of systemic inflammation, platelet activation, and myocardial necrosis. J Am Coll Cardiol. 2005;45(2):229-237.

46. Lodh M, Goswami B, Parida A, Patra S, Saxena A. Assessment of serum leptin, pregnancy-associated plasma protein A and CRP levels as indicators of plaque vulnerability in patients with acute coronary syndrome. Cardiovasc J Afr. 2012;23(6):330-335.

47. Persico MG, Vincenti V, DiPalma T. Structure, expression, and receptor-binding properties of placenta growth factor (PIGF). Curr Top Microbiol Immunol. 1999;237:31-40.

48. Khurana R, Moons L, Shafi S, et al. Placental growth factor promotes atherosclerotic intimal thickening and macrophage accumulation. Circulation. 2005;111(21):2828-2836.

49. Roncal C, Buysschaert I, Gerdes N, et al. Short-term delivery of anti-PIGF antibody delays progression of atherosclerotic plaques to vulnerable lesions. Cardiovasc Res. 2010;86(1):29-36.

50. Heeschen C, Dimmeler S, Fichtlscherer S, et al. Prognostic value of placental growth factor in patients with acute chest pain. JAMA. 2004; 291(4):435-441.
51. Lenderink T, Heeschen C, Fichtlscherer S, et al. Elevated placental growth factor levels are associated with adverse outcomes at four-year follow-up in patients with acute coronary syndromes. J Am Coll Cardiol. 2006;47(2):307-311.

52. Apple FS, Pearce LA, Chung A, Ler R, Murakami MM. Multiple biomarker use for detection of adverse events in patients presenting with symptoms suggestive of acute coronary syndrome. Clin Chem. 2007; 53(5):874-881.

53. Möckel M, Danne O, Müller R, et al. Development of an optimized multimarker strategy for early risk assessment of patients with acute coronary syndromes. Clin Chim Acta. 2008;393(2):103-109.

54. Glaser R, Peacock WF, Wu AH, Muller R, Möckel M, Apple FS. Placental growth factor and B-type natriuretic peptide as independent predictors of risk from a multibiomarker panel in suspected acute coronary syndrome (Acute Risk and Related Outcomes Assessed With Cardiac Biomarkers [ARROW]) study. Am J Cardiol. 2011;107(6): 821-826.

55. Van Mieghem CA, Bruining N, Schaar JA, et al. Rationale and methods of the integrated biomarker and imaging study (IBIS): combining invasive and non-invasive imaging with biomarkers to detect subclinical atherosclerosis and assess coronary lesion biology. Int J Cardiovasc Imaging. 2005;21(4):425-441.

56. Prasad A, Tsimikas S. Candidate biomarkers for the detection of coronary plaque destabilization and rupture. Curr Atheroscler Rep. 2008;10(4):309-317.

57. Lobbes MBI, Kooi ME, Lutgens E, et al. Leukocyte counts, myeloperoxidase, and pregnancy-associated plasma protein A as biomarkers for cardiovascular disease: towards a multi-biomarker approach. Int J Vasc Med. 2010;2010:726207.

58. Schaub N, Reichlin T, Meune C, et al. Markers of plaque instability in the early diagnosis and risk stratification of acute myocardial infarction. Clin Chem. 2012;58(1):246-256.
Research Reports in Clinical Cardiology

\section{Publish your work in this journal}

Research Reports in Clinical Cardiology is an international, peerreviewed, open access journal publishing original research, reports, editorials, reviews and commentaries on all areas of cardiology in the clinic and laboratory. The manuscript management system is completely online and includes a very quick and fair peer-review system.

\section{Dovepress}

Visit http://www.dovepress.com/testimonials.php to read real quotes from published authors. 